\author{
F. Rubulotta \\ D. C. Scales \\ S. D. Halpern
}

\section{Night shifts, human factors, and errors in the ICU: a causal pathway?}

Received: 16 November 2015

Accepted: 17 November 2015

Published online: 7 December 2015

(C) Springer-Verlag Berlin Heidelberg and ESICM 2015

F. Rubulotta (

Centre for Perioperative Medicine and Critical Care Charing Cross Hospital Intensive Care Unit, Imperial College NHS Trust, Fulham Palace Road, W6 8RF London, UK

e-mail: frubulotta@hotmail.com; francesca.rubulotta@imperial.nhs.uk

Tel.: +447540500674

D. C. Scales

Interdepartmental Division of Critical Care and Department of Medicine, University of Toronto, Sunnybrook Health Sciences

Centre, Toronto, Canada

e-mail: damon.scales@sunnybrook.ca

S. D. Halpern

Perelman School of Medicine at the University of Pennsylvania, Philadelphia, USA

e-mail: shalpern@exchange.upenn.edu

Preventable medical errors continue to occur despite increased worldwide awareness and resources devoted to improve the safety of health systems. Factors that influence a clinical team's effectiveness include individual team members' performance, safety and quality of medical equipment, organization and structure of each ward, patient population, and the underlying institutional culture [1]. Human factors (ergonomics) is the study of human interactions with tools, devices, and systems with the goal of enhancing safety, efficiency, and user satisfaction [1,2].

For many years, several high-hazard industries have recognized the importance of minimizing the risk from human errors [3]. For example, the nuclear sector has led the way in understanding, measuring, and improving human reliability, resulting today in a very safe industry [3, 4]. The adoption of a human factors approach in aviation has improved the design of traffic control, aircraft interiors, and pilot working hours [4]. By contrast, quality and safety initiatives in health services have tended to focus on quantitative measurement and casual explanations of adverse events and incidents, with the primary method being root cause analysis (RCA) [3, 4]. The use of ergonomics in medicine, such as workplace domain analysis, might lead in the future to more adequate process improvements in the delivery of innovative health care, and in particular intensive care medicine [3, 4]. Health care providers and managers must carefully consider the impact of the many "performance-shaping factors" that are known to play a role in human errors to understand how best to optimize health care settings.

In this context, Maltese and colleagues are to be commended for investigating the relationship between doctors' cognitive performance during nighttime shift work and the risk of medical errors [5]. Cognitive skills were tested according to the Wechsler adult intelligence scale and the Wisconsin card sorting test showing that four cognitive abilities worsened after a night shift: working memory capacity, speed of processing information, perceptual reasoning, and cognitive flexibility. This work complements an extensive literature showing the adverse effects of sleep deprivation and fatigue on an individual clinician's performance [6-8]. Sleep loss most acutely degrades performance on tasks requiring vigilance, cognitive skills, verbal processing, and complex problem solving [7-10]. In the early morning hours, after nearly $24 \mathrm{~h}$ without sleep (e.g., at the end of difficult on-call shift), psychomotor performance can be impaired "to an extent equivalent to or greater than is currently acceptable for alcohol intoxication" [11]. These studies have contributed to the implementation of work hour limits for clinicians in training [6-14].

The approach selected by Maltese and colleagues is innovative given the focus on the measurement of cognitive performance, which might be a marker for "near misses," 
instead of actual incidents. This is a crucial shift in the management of human errors. They did not seek to determine how many errors were attributable to night work, but rather focused on what can be modified in efforts to prevent errors, and in whom. In this regard, the authors' two key results are that the cognitive abilities of ICU physicians consistently decreased after a night shift, and that these effects were similar among residents and attending physicians. An additional important finding is that, contrary to prior belief, a few hours of sleep at night do not improve most categories of cognitive performance.

This approach contributes to the underlying science by suggesting possible mechanisms of human error, but also has limitations. Specifically, degradations in psychomotor performance are not the same as degradations in clinical skill. And even degradations in clinical skill do not neatly translate into demonstrable patient harm. This is particularly true in the context of critical care delivery, in which the multidisciplinary and team-based approach to care may mitigate any impact of an individual's worsened psychomotor performance on patients.

Thus, the current study contrasts, for example, with the recent study by Parshuram and colleagues [15] evaluating the effects of three resident schedules in the ICU on patient safety, resident well-being, and continuity of care. This study showed no effects of residents' schedules on adverse events, residents' sleepiness in the daytime or at night, or patient mortality. The finding that residents' somatic symptoms were more severe and more frequent with longer schedules, and ICU staff rated residents' knowledge and decision-making as worse with an intermediate schedule, suggests that such intermediate endpoints may not translate into clinical events.

Also relevant to the current work are the several studies showing that as long as there are some physicians in the ICU at night (even trainees), patient outcomes are not improved by adding more (better rested) physicians or more senior physicians [12-14]. The fact that adding rested physicians does not clearly improve upon the care that tired physicians are providing suggests that degradations in cognitive and psychomotor performance may not directly translate into patient harm in the context of team-based ICU care.

In conclusion, human factors research in the complex health care system is poorly utilized and understood. Qualitative analysis of possible human errors and near misses might improve quality of care delivery and patient safety. We still have much to learn about how best to structure teams and schedules to deliver critical care in ways that best promote patient-centered outcomes. Night shifts are likely to remain an essential component of critical care delivery, and describing the impact of sleep deprivation on clinicians' performance is essential to design safer health systems. However, work along the spectrum of science, from human factors all the way to outcomes assessment, will be crucial in achieving this important goal.

\section{References}

1. Lundstrom T, Pugliese G, Bartley J, Cox J, Guither C, Michigan D, Illinois O (2002) Organizational and environmental factors that affect worker health and safety and patient outcomes. Am J Infect Control 30:93-106

2. Wears RL, Perry SJ (2002) Human factors and ergonomics in the emergency department. Ann Emerg Med 40:206-212

3. Cassin B, Barach P (2012) Making sense of root cause analysis investigations of surgery-related adverse events. Surg Clin North Am 92:101-115. doi: 10.1016/j.suc.2011.12.008

4. Amalberti R, Auroy Y, Berwick D, Barach P (2005) Five system barriers to achieving ultra-safe health care. Ann Intern Med 142:756-764

5. Maltese F, Adda M, Bablon A, Hraeich S, Guervilly C, Lehingue S, Wiramus S, Leone M, Martin C, Vialet R, Thirion X, Roch A, Forel JM, Papazian L (2015) Night shift decreases cognitive performance of ICU physicians. Intensive Care Med. doi: 10.1007/s00134-015-4115-4
6. Weinger M, Englund C (1990) Ergonomic and human factors affecting anesthetic vigilance and monitoring performance in the operating room environment. Anesthesiology 73:995-1021

7. Weinger MB, Ancoli-Israel S (2002) Sleep deprivation and clinical performance. JAMA 287:955-957

8. Howard SK, Rosekind MR, Katz JD, Berry AJ (2002) Fatigue in anesthesia. Anesthesiology 97:1281-1294

9. Gaba DM, Howard SK (2002) Fatigue among clinicians and the safety of patients. N Engl J Med 347:1249-1255

10. Veasey S, Rosen R, Barzansky B, Rosen I, Owens J (2002) Sleep loss and fatigue in residency training: a reappraisal. JAMA 288:1116-1124

11. Dawson D, Reid K (1997) Fatigue, alcohol and performance impairment. Nature 388:235

12. Kerlin MP, Small DS, Cooney E, Fuchs BD, Bellini LM, Mikkelsen ME, Schweickert WD, Bakhru RN, Gabler NB, Harhay MO, Hansen-Flaschen J, Halpern SD (2013) A randomized trial of nighttime physician staffing in an intensive care unit. N Engl J Med 368:2201-2209
13. Kerlin MP, Harhay MO, Kahn JK, Halpern SD (2015) Nighttime intensivist staffing, mortality, and limits on life support: a retrospective cohort study. Chest 147(4):951-958

14. Wallace DJ, Angus DC, Barnato AE, Kramer AA, Kahn JM (2012) Nighttime intensivist staffing and mortality among critically ill patients. N Engl J Med 366:2093-2101

15. Parshuram CS, Amaral AC, Ferguson ND, Baker GR, Etchells EE, Flintoft V, Granton J, Lingard L, Kirpalani H, Mehta S, Moldofsky H, Scales DC, Stewart TE, Willan AR, Friedrich JO, Canadian Critical Care Trial Group (2015) Patient safety, resident wellbeing and continuity of care with different resident duty schedules in the intensive care unit: a randomized trial. CMAJ 187(5):321-329 